VII International Congress of

Science, Technology,

Entrepreneurship and

Innovation (SECTEI 2020)

Corresponding Author:

R. Villacrés

rvillacres@espoch.edu.ec

Published: 26 August 2021

Production and Hosting by

Knowledge E

(c) R. Villacrés et al. This article is distributed under the terms of the Creative Commons Attribution License, which permits unrestricted use and redistribution provided that the original author and source are credited.

\section{Open Innovation Experiences Developed in the Hub Center Project for the Solution of High Impact Problems of the Central Region of the Country}

\section{Experiencias de Innovación Abierta Desarrol- ladas en el Proyecto Hub Centro para la Solución de Problemáticas de Alto Impacto de la Región Centro del País}

\author{
R. Villacrés, P Arguello, and E. Pullutaci
}

Carrera de Administración de Empresas, Facultad de Administración de Empresas, Escuela Superior Politécnica de Chimborazo, Riobamba, Ecuador

\section{Abstract}

This article allows us to externalize the experiences mainly of the challenges of open innovation developed in the cities of Riobamba, Latacunga and Ambato, understood these cities as the HUB's area of influence, in addition, to refer to the current situation as organizations that promote solutions to these problems existing ones that have been confronted taking innovation as the main instrument to provide solutions. The main objective of this review article is to refer to the open innovation experiences that the HUB Center has carried out at the time of its management, specified in the development of the document. The development of this work was done through the exploration of information from authors who have already treated these issues with another perspective and with other ways of doing things, with enough experience in their field, also the deductive study method allows to go from a general context to a specific one, and according to the requirements that this research estimates. The HUB Center is a networked space with 8 Higher Education Institutions plus the Ecuadorian Corporation for Research Development and the Academy that are members of this network, through these institutions and with the articulation of the HUB, local development is promoted from the academy to the productive and social sectors supported by the central government and the local governments of each province.

Keywords: HUB Center, open innovation, high impact problems, higher education institutions, zone 3, innovation challenges.

\section{Resumen}

Este articulo permite exteriorizar las experiencias principalmente de los retos de innovación abierta desarrollados en las ciudades de Riobamba, Latacunga y Ambato, entendidas estas ciudades como la zona de influencia del HUB, además, referir la situación actual en cuanto organizaciones que promuevan soluciones a esas problemáticas existentes que se han afrontado tomando a la innovación como principal instrumento para dar soluciones. El objetivo principal de este artículo de revisión es referir las experiencias de innovación abierta que el HUB Centro ha realizado en el tiempo de su gestión, especificadas en el desarrollo del documento. El desarrollo de este trabajo se lo realizo mediante la exploración de información 
de autores que ya han tratado estos temas con otra perspectiva y con otras formas de hacer las cosas, con una experiencia basta en su campo, asimismo el método de estudio deductivo permite ir de un contexto general a uno concreto, y de acuerdo con los requerimientos que esta investigación estima. EI HUB Centro es un espacio que funciona en red con 8 Instituciones de Educación Superior más la Corporación Ecuatoriana para el Desarrollo de la Investigación y la Academia que son miembros de esta red, mediante estas instituciones y con la articulación del HUB se promueven el desarrollo local desde la academia hacia los sectores productivos y sociales apoyados por el gobierno central y los gobiernos locales de cada provincia.

Palabras Clave: HUB Centro, innovación abierta, problemas de alto impacto, instituciones de educación superior, zona 3, retos de innovación.

\section{Introducción}

Mucho se ha dicho respecto a la innovación abierta en los tiempos contemporáneos, a partir del uso frecuente del término en la literatura común. Desde su aparición la innovación abierta ha estado asociada a la generación de nuevos conocimientos de forma colaborativa con los agentes externos a una organización, pero motivado por la realidad mundial conocida hoy en día la innovación abierta se emplea, además en buscar soluciones a problemas de manera novedosa, promoviendo la creatividad de los actores que intervienen. La innovación abierta es un camino hacia la solución de necesidades de manera conjunta que surgen constantemente en las personas. El HUB de Innovación y Transferencia de Tecnología Centro es un espacio de red destinado a promover la innovación, el emprendimiento y la transferencia de tecnología apoyado por sus miembros que son las instituciones de educación superior de la zona 3 del país, esta organización impulsa el fortalecimiento de los ecosistemas innovadores basados en el conocimiento y apoyados en la suma de esfuerzos de las instituciones de educación superior que forman parte de la zona. En la zona de influencia del HUB ITT Centro tenemos tres ciudades: Riobamba, Ambato y Latacunga que viven problemas sociales, de desarrollo, económicos, académicos entre otros, éstos son conocidos como problemas de un impacto superior o de alto impacto, estos problemas han tratado de ser solucionados por los diferentes gobernantes de turno ya sean nacionales o locales, y en algunos casos por organización internacionales dedicadas al desarrollo de poblaciones latinoamericanas, pero se conoce que en los últimos tiempos estos problemas han ido en decadencia hacia una realidad mucha más difícil y sin organizaciones que promuevan soluciones reales, estos problemas gigantescos formaran parte de una realidad sin remedio. Es por ello por lo que como autores de este articulo creemos necesario compartir las acciones que se están llevando a cabo para mejorar aquella 
realidad a través del ingenio y el talento capaz que existe en la zona centro del país y con conocimiento propio de las necesidades reales.

\section{Metodología}

El tema que vamos a tratar sugiere una metodología de estudio exploratoria, en vista de que el tipo de investigación que se realizara pretende abordar a la innovación abierta desde una perspectiva acorde a la realidad de la zona centro del país. Además, la metodología que da soporte al tipo de estudio citado con anterioridad es la deductiva debido a que se abordan temas generales, regionales, nacionales y locales de la temática en mención.

\subsection{Exploratorio}

Los estudios exploratorios se realizan cuando el objetivo es examinar un tema o problema de investigación poco estudiado, del cual se tienen muchas dudas o no se hayan abordado antes.

\subsection{Deductivo}

La metodología deductiva es una destreza de raciocinio que se utiliza para deducir conclusiones racionales a una vez se haya efectuado una serie de antecedentes o principios.

\section{Desarrollo y Discusión}

\subsection{Desarrollo}

\subsubsection{Innovación}

El concepto de innovación ha evolucionado juntamente con la historia de los avances científicos y tecnológicos de la humanidad y en esencia hace referencia a un proceso mental cuyo resultado creativo desarrolla un nuevo producto, diseño, proceso o modelo cuyo propósito es agregar valor a la organización. Consecuentemente, como lo define Palaci la innovación en líneas generales consiste en utilizar conocimiento para construir un nuevo camino que lleve a una determinada meta [1].

Al hacer una aproximación histórica a propósito del tema que nos convoca en esta investigación, observamos que, según Avendaño and William el concepto de innovación 
tiene su origen en el desarrollo de nuevas ideas, las cuales sumadas al elemento valor marcan la diferencia con la creatividad como mera capacidad [2]

A medida que el termino de innovación ha ido formando parte de la literatura común, se ha establecido el alcance del concepto según Albornoz en donde menciona a la innovación como la capacidad de asumir los cambios y desarrollar capacidades creativas [3].

La innovación se ha identificado principalmente con el sector empresarial debido a que este término ha sido fundamental para que las empresas tomen a la innovación como su motor para el crecimiento y el desarrollo de sus capacidades de mejora en un ambiente competitivo. Por consiguiente, Anllo et al. explican que la innovación es la introducción de un nuevo o significativamente mejorado producto (bien o servicio), de un proceso, de un nuevo método de comercialización o de un nuevo método organizativo, en las prácticas internas de la empresa, la organización del lugar de trabajo o las relaciones exteriores [4].

A medida que el desarrollo humano avanza se logran perfeccionamientos no solo en nuevas tecnologías tangibles e intangibles para su beneficio, sino también en nuevos conceptos que permiten el progreso intelectual de las personas, en ese contexto la Cámara de Comercio de España expresa a la innovación como la definición de nuevos procedimientos para sistematizar ciertas operaciones (compras, control de calidad, seguridad en el trabajo, etc.), modificación de las formas de relacionarse con clientes y proveedores incorporando nuevas tecnologías de comunicación, redefiniendo las estrategias de comercialización de productos o servicios, etc. [5].)

Las mejores innovaciones están apoyadas en las experiencias que aportan los clientes o consumidores, basado en una empatía que nace con una idea, un determinado producto o un servicio que una persona decide que lo necesita, o simplemente en una solución novedosa de un problema. En ese sentido según Innovatec la Innovación se considera como sinónimo de producir, asimilar y explotar con éxito una novedad, en las esferas económicas y sociales, de forma que aporte soluciones inéditas a los problemas y permita así responder a las necesidades de las personas y de la sociedad [6].

Cuando nos referimos a la innovación se debe tener presente siempre que, si no existen personas comprometidas con el cambio hacia una mentalidad de superación y colaboración, las opiniones que estas expresen no serán completamente reales debido a un enfoque de su realidad distinta a lo que realmente sucede, es por ello que como lo expresa Chávez la innovación, se refiere tanto a la gestión como a la tecnología [7]. Una empresa que innova es una empresa que también es capaz de aprender. Los métodos de organización y gestión son cruciales para aprender: fijar y supervisar los objetivos; vincular los procedimientos de recursos humanos y los incentivos con las metas, y obtener la opinión de los trabajadores acerca de cómo seguir mejorando [7]. 
La innovación desde un enfoque empresarial según lo explican Anllo et al. es aquel que transforma las ideas en inventos y los inventos en productos rentables y comercializables, demarcando territorios entre los inventos (una mera idea nueva) y las innovaciones (la introducción comercial exitosa de dicha idea) [4]. Todo giraba en torno a los innovadores geniales que hicieron una sola cosa de la producción y la tecnología, comprendiendo que la clave del éxito pasaba por la diferenciación de producto y/o proceso [4].

El internet de las cosas es por supuesto innovación que supone el progreso y futuro del desarrollo mundial, entonces es así que, según Álvarez Rivero el concepto abarca la idea de que, cada vez más, dispositivos físicos diversos estarán en red, cambiando informaciones entre sí sin la intervención humana. Sin duda la innovación desde un punto de vista tradicional [8].

\subsubsection{Tipos de innovación}

La innovación se relaciona generalmente con una inspiración momentánea o una idea que surge de pronto, todos estos elementos suman a un proceso que permite la creación de buenas ideas, y producto de ellas se generan nuevos bienes, productos y/o servicios o en su defecto mejorados, que constituyen la apertura de nuevos mercados y la consolidación de ya existentes. Además, la innovación se trata también de modificar las formas de hacer las cosas o de modificar la percepción de un concepto establecido con anterioridad. De la misma manera existen varias clasificaciones en cuanto a los tipos de innovación, para este trabajo de investigación tomamos las consideradas con mayor relevancia con su significado correspondiente, la innovación del producto, innovación de servicios, business model innovation, innovación de procesos y tecnología, innovación organizativa, innovación social, innovación radical, innovación incremental, innovación sostenible, innovación cerrada e innovación abierta siendo esta ultima el tema central de la investigación, en este sentido, un autor significativo es Zapfl quien menciona la siguiente clasificación [9]:

1. Innovación del producto: Los productos se refieren tanto a productos materiales como a servicios intangibles, como los servicios que satisfacen las necesidades de los clientes y que, por lo tanto, son adquiridos por ellos. Con las innovaciones de los productos, una empresa gana dinero y trata de diferenciarse de la competencia.

2. Innovación de servicios: Las innovaciones de servicios son como las innovaciones de productos cuando se trata de venderlos directamente al cliente, por ejemplo, seguros o consultoría de gestión. Incluso si los servicios no se venden activamente, como en el caso de las empresas de fabricación, cada empresa sigue prestando servicios a sus clientes, por ejemplo, en logística, reclamaciones, asesoramiento 
de ventas, etc., incluso si no se venden activamente. Aquí es donde también entra la innovación cuando se trata de la diferenciación y el entusiasmo de los clientes.

3. Business model innovation: El modelo de negocio es la forma en que una empresa funciona y gana dinero. La innovación del modelo de negocio abarca innovaciones en estrategia, marketing, cadenas de suministro, creación de valor, precios o estructuras de costes.

4. Innovación de procesos y tecnología: Como su nombre indica, se trata de innovaciones tecnológicas, como la creación de productos y servicios. En principio, también son innovaciones de proceso. Estos incluyen, por ejemplo, procesos de producción o tecnologías de TI para aplicaciones. Las innovaciones de productos, las mejoras de calidad o el ahorro de costes a menudo van de la mano con las innovaciones tecnológicas y de procesos.

5. Innovación organizativa: Las innovaciones organizacionales afectan el proceso y la estructura organizacional. Éstas pueden ser innovaciones en los procesos de la organización o en la gestión, por ejemplo, nuevas herramientas para medir la satisfacción del cliente u optimizar los procesos de entrega para reducir costes.

6. Innovación social: Las innovaciones sociales son innovaciones en las que el beneficio recae en la sociedad y el propósito no es principalmente el lucro. Algunos ejemplos son la innovación en la educación, la reducción de la pobreza, la igualdad de oportunidades y la salud.

7. Innovación radical: Son nuevos productos, servicios o procesos y suponen un cambio e innovación significativos. En consecuencia, el impacto es también mayor - por ejemplo, se pueden crear nuevos mercados como resultado de ello.

8. Innovación incremental: Es la optimización y el desarrollo posterior de productos, servicios o procesos existentes. El objetivo y los beneficios son la optimización del beneficio para el cliente, la reducción de costes, el reposicionamiento, la adaptación para la introducción en nuevos mercados o la adaptación a nuevas circunstancias, como nuevas leyes y normas.

9. Innovación sostenible: La preservación o innovación continua se refiere a la mejora de las innovaciones existentes, similar a las innovaciones incrementales. Este tipo de innovación se centra en los clientes actuales y sus necesidades.

10. Innovación cerrada: Implica solo recursos internos para generar ideas, desarrollar e implementar innovaciones.

11. Innovación abierta: Integra en el proceso de innovación a socios externos como clientes, institutos de investigación o proveedores. 


\subsubsection{Innovación abierta}

En la actualidad la innovación abierta se trata de una corriente adoptada por muchas empresas a nivel mundial debido a que este tipo de innovación se basa en la colaboración de las personas y entidades externas a una organización. Pero para que este tipo de innovación forme parte del entorno organizacional previamente exige acceder a la co - creación con profesionales u organizaciones externas a la entidad, este trabajo colaborativo se entiende a través de convenios, alianzas, congresos, cátedras de investigación superior, retos de innovación abierta o ecosistemas de innovación, además la organización deberá estar dispuesta a compartir los beneficios de aquella colaboración. En ese contexto según Ramírez and García Peñalvo la ciencia abierta, como bien común, abre posibilidades para el desarrollo de las naciones a través de innovaciones y construcciones colaborativas que ayudan a democratizar el conocimiento [10]. Los avances en la materia aún son incipientes y el triángulo ciencia abierta, co-creación del conocimiento e innovación abierta se presenta como una oportunidad de generar un aporte original, desde la investigación, para la teoría y las prácticas educativas abiertas [10].

La innovación abierta supone alianzas estratégicas que permiten el desarrollo de cada parte interesada e involucrada, puesto que este tipo de innovación según Benavente and Suaznábar involucra a grandes empresas y startups llevando adelante procesos de co-creación y colaboración para poder innovar al ritmo que les impone el mercado, con soluciones más baratas y ágiles [11].

La innovación abierta resulta ir más allá que solo un método para generar cosas nuevas o mejoradas, ésta es una estrategia que permite tomar a la innovación en un campo muy amplio y que no sea la frontera entre lo que se puede hacer y una quimera en los límites organizacionales o áreas de I+D, sino que como lo dicen López Rodríguez and García Lorenzo implica una filosofía de gestión de la innovación basada en la articulación de un proceso de colaboración y participación con otros agentes, lo cual requiere de la elaboración de acuerdos que contemplen, además del reparto de costes, el reparto de la propiedad de los resultados [12].

Cuando se habla de generar conocimiento la innovación abierta resulta ser un camino hacia ese resultado, la gestión del conocimiento supone transferir el conocimiento desde el lugar en el que se genera hacia el lugar en el cual se va a emplear, entonces la innovación abierta también permite generar conocimiento en colaboración entre varias organizaciones que trabajan colaborativamente para su bien común y esto ayuda a evitar que las empresas u organizaciones se encierren en sí mismos, puesto que según lo cita Álvarez Aros and Bernal Torrez es una estrategia que permite incorporar conocimiento, experiencias o tecnologías que contribuyen a mejorar tanto los productos 
y procesos, las actividades organizacionales y comerciales y que, integra la inteligencia colectiva en la búsqueda del conocimiento externo con clientes, proveedores, intermediarios, centros de investigación, instituciones educativas y competidores para potenciar la capacidad innovadora y competitiva de las empresas y las economías [13].

El entorno que rodea a las organizaciones está integrado por una diversidad de recursos que en su conjunto permitirían el desarrollo significativo de organizaciones que buscan sobrepasar los límites de sus capacidades en beneficio de sus grupos de interés internos y como resultado de la innovación abierta, también de sus grupos de interés externos, entonces según Obea Research Group la innovación abierta significa que las organizaciones pueden hacer uso de recursos externos y de las mejores prácticas para complementar el valor de sus propios activos de innovación, obteniendo mayor retorno de la inversión [14].

Ahora bien según lo define BBVA la innovación abierta permite el uso de los flujos internos y externos de conocimiento para acelerar la innovación interna y ampliar los mercados para el uso externo de dicha innovación [15], además este tipo de innovación acelera la misma, reduce costes y riesgos asociados a un determinado giro empresarial, sin olvidar que el conocimiento generado deja de ser unidireccional y permite enriquecer a todos los asociados.

Estos términos principalmente IA conjugan con la forma creativa de cada persona en la que ve una situación adversa como una oportunidad de aprovechamiento de aquel estado y la convierte en recursos, puesto que, según Pombo la innovación abierta utiliza recursos externos e internos, compartiendo ideas, conocimientos, riesgos y beneficios, en un ambiente de colaboración global, cuyo principal objetivo es acelerar el proceso de innovación [16].

La parte que mejor se puede obtener beneficios de fuentes externas suponen una ventaja realmente buena para las empresas debido a que éstas ayudan a obtener ventajas competitivas sobre su competencia en el mercado, ya que según Sánchez la Innovación Abierta es un paradigma que asume que las empresas pueden y deben apalancarse de ideas provenientes de fuentes externas e internas mientras buscan desarrollar un mayor avance tecnológico [17].

\subsubsection{Retos de innovación}

Los retos por su definición suponen una acción complicada de llevar a cabo. Las organizaciones que promueven este tipo de certámenes cuentan con incentivos de diversos tipos para retribuir el esfuerzo a los ganadores, de ahí que el reto según Alba es un objetivo o empeño difícil de llevar a cabo, y que constituye por ello un estímulo y un desafío para quien lo afronta [18]. 
Dar solución a una problemática de una manera innovadora y libre para solucionarla, se ha convertido en una forma de generar conocimiento porque a través de la ideación de estas soluciones se crea nuevas formas de hacer las cosas y la mayoría de estas soluciones dan pie a maneras poco comunes de afrontar los problemas, en vista de ello como lo expresa Clos los retos de innovación son problemas para las cuales se necesita y se busca activamente soluciones creativas que permitan resolverlos [19]. En general, los retos de innovación se mueven en entornos de incertidumbre, es decir, el grado de conocimiento adquirido alrededor del problema es reducido, o muy elevado hasta llegar a un punto de saturación a partir del cual es necesario buscar nuevas vías para solucionarlo [19].

Al encarar un problema y buscar una solución se generan muchas ideas de cómo resolverlos, pero el beneficio de realizar los retos de innovación es que estos permiten documentar las formas en las que se resolverían generando así, nuevas herramientas para el progreso intelectual de las personas a través del desarrollo de las capacidades de quienes deciden afrontarlos, ya que según el Centro de Ciencia y Tecnología de Antioquia los retos de innovación consiste en un ejercicio de identificación de soluciones a problemas, necesidades y oportunidades en las empresas, clientes o anclas, que han participado o están participando en el programa, por parte de otras empresas de la ciudad en temas relacionados, por ejemplo, con la modernización de sus procesos o su tecnología, solución de problemas para acceder a nuevos mercados, desarrollo de nuevos productos, sustitución de importaciones, entre otros; donde la innovación y el desarrollo tecnológico sean el factor que permita mejorar la competitividad y fortalecer lazos de confianza entre ambas empresas [20].

Además de las iniciativas ya citadas en el Ecuador existen entidades como las de gobierno que promueven los retos de innovación abierta en post de colaborar con el desarrollo nacional. En ese contexto según la SENESCYT, el Ministerio de Producción, Comercio Exterior, Inversiones y Pesca (Mpceip) y la Secretaría de Educación Superior, Ciencia, Tecnología e Innovación (SENESCYT), junto a la Fundación Consorcio de Queseras Rurales Comunitarias, se realizó primer reto de innovación agroindustrial con el apoyo de la Red Cedia y la Alianza para el Emprendimiento e Innovación (AEI), bajo la iniciativa de Fundación Consorcio de Queseras Rurales Comunitarias de la empresa El Salinerito [21].

\subsubsection{Experiencia de innovación abierta en Ecuador}

En el país existen pocas organizaciones que se dedican al fomento de la investigación, el desarrollo y la innovación (l+D+i). La Corporación Ecuatoriana para el Desarrollo de la Investigación y la Academia es una de esas organizaciones que, basadas en su filosofía de generar desarrollo en colaboración con la academia, el sector empresarial 
y el gobierno busca el beneficio para la sociedad, el sector productivo y en general para el país. Según CEDIA el Reto Empresarial es uno de sus programas que ofrecen a sus miembros [22]: El proceso comienza cuando una empresa identifica una necesidad que debe ser subsanada y considera que la solución puede provenir de un actor asociado al ecosistema. En caso de que la empresa no ha logrado definir de manera clara la problemática, CEDIA tiene expertos para la realización de talleres en los cuales una empresa puede definir y priorizar problemáticas que pueden ser resueltas a través de Retos de innovación abierta. Con la descripción del problema, CEDIA organizará una convocatoria a las IES asociadas al ecosistema invitándoles a que presenten sus propuestas de solución. Con la participación de un comité evaluador que se designará para el efecto, se escogerán las mejores propuestas las que finalmente serán analizadas en forma conjunta entre CEDIA y la empresa interesada en dar solución a su problema, lo que llevará a la selección de una propuesta ganadora. En caso de que las condiciones bajo las cuales se planteó la convocatoria contemplen que el proceso debe continuar con la implementación de la solución propuesta, se pasará a la fase de ejecución de esta; en caso contrario, se otorgará un premio a la IES autora de la propuesta ganadora, con lo que concluirá el proceso [22].

El gobierno central a través de la Secretaria de Educación Superior Ciencia, Tecnología e Innovación - SENESCYT impulsa el desarrollo de sus poblaciones, es por ello que genera este tipo de alianzas (CEDIA) para que sean éstos los entes articuladores entre el gobierno y la academia, conjuntamente con el sector empresarial para impulsar soluciones a problemas que aquejan a sus ciudadanos, en ese contexto según CEDIA impulsa el reto de innovación abierta: Solución a problemas de transporte público de la ciudad de Riobamba [23]

Esta ciudad fue sede de la Semana de la Ciencia, Tecnología, Emprendimiento e Innovación, importante actividad organizada por la Municipalidad de Riobamba con el apoyo de la Universidad Nacional de Chimborazo y la Escuela Superior Politécnica de Chimborazo, varias instituciones públicas y empresas privadas de la ciudad con el apoyo de CEDIA [23].

Al igual que, en la problemática identificada en la ciudad de Riobamba, el reto en la ciudad de Latacunga se impulsa con éxito para dar solución a una problemática que no solo es cuestión de las grandes urbes. Se sabe que la congestión vehicular colabora a la decadencia de la salud de las personas es por ello por lo que según la SENESCYT se realizara el reto de innovación [24]: Fomento al uso de medios alternativos de transporte en la ciudad de Latacunga promueve una solución innovadora.

La Universidad de las Fuerzas Armadas, ESPE, la SENESCYT y CEDIA convocaron a la ciudadanía en la zona a presentar incitativas con potencial de alto impacto respecto al fomento del uso de medios alternativos de transporte público masivo en la ciudad de Latacunga [25]. 
Además de CEDIA, en el país existe otra organización internacional con sede en Quito que promueve el desarrollo, entonces según la (Agencia de Promociones Económicas CONQUITO) Empretec es un programa internacional de la Organización de Naciones Unidas (ONU), que ha sido concebido y diseñado para identificar, formar y apoyar a emprendedores.

Se encuentra administrado por la Secretaría de la UNCTAD (Conferencia de las Naciones Unidas para el Comercio y Desarrollo) en Ginebra (Suiza). Este programa ha impulsado un cambio de comportamiento a emprendedores de 40 diferentes países en el mundo, adaptándose a la realidad local en cada uno de ellos. En Ecuador, CONQUITO es el Centro Nacional Empretec [26].

En la zona 3 del país existe la organización Corporación Civil para el Desarrollo Económico de Ambato y Tungurahua dedicada al apoyo de emprendedores que están emergiendo al mercado y requieren de expertos que ayuden a consolidar su negocio o idea de negocio [27]. CorpoAmbato impulsa el desarrollo productivo de la ciudad y la provincia, a través de la articulación de proyectos enmarcados en la Agenda de Competitividad de Tungurahua, para que así aporten al desarrollo de la Matriz Productiva del país [27].

Los retos de innovación van enfocados a varios sectores productivos y muestra de ello es el reto que se planteó para el aprovechamiento de todos los recursos asociados a la producción de leche organizado por el Ministerio de Producción, Comercio Exterior, Inversiones y Pesca con el CIL Centro de la Industria Láctea con el apoyo de CEDIA y la $\mathrm{AEI}$, quienes proponen el reto al desarrollo de alternativas para el aprovechamiento del lacto suero líquido de la industria láctea formal ecuatoriana, para el desarrollo de nuevos productos alimenticios de consumo humano final que no existan en el mercado ecuatoriano [28].

\subsubsection{Innovación abierta: Realidad del Hub ITT Centro}

El proyecto HUB de ITT (innovación y transferencia de tecnología), nace como una iniciativa de la Secretaria de Educación Superior, Ciencia, Tecnología e Innovación SENESCYT, para impulsar el desarrollo de las actividades de innovación, emprendimiento y transferencia de tecnología llevadas a cabo en las Instituciones de Educación Superior (IES). Generando un ecosistema que permite el desarrollo y ejecución de proyectos de innovación y emprendimiento con enfoque productivo, a través de la articulación entre el sector académico, empresarial y gobierno. Promoviendo así, el desarrollo de la economía basada en el conocimiento y la institucionalización de los procesos de innovación, emprendimiento y transferencia de tecnología. 
Es un espacio en Red destinado a fomentar la innovación, el emprendimiento y la transferencia de conocimiento y tecnología desde las Instituciones de Educación Superior (IES) que forman parte de la Zona 3, de tal manera que se fortalezca el ecosistema de innovación, aportando así al desarrollo productivo del país y potencializando la economía basada en el conocimiento.

\subsection{Discusión de Resultados}

\subsubsection{Discusión}

En el desarrollo de la presente investigación se evidencia a la innovación abierta como una forma de trabajo conjunto entre varios actores que pretenden encontrar formas diferentes e innovadoras de promover acciones a adversidades que están sucediendo en el entorno próximo de cada individuo dispuesto a romper esquemas para acceder a escenarios de conocimientos que permiten abordar aquellas adversidades con mejores argumentos e ideas de solución.

En ese contexto, el HUB ubicado en la zona centro del país busca ser la organización eje entre los sectores productivos, los entes de gobierno, la academia y la sociedad civil, para promover y fortalecer los espacios que generen innovación y desarrollo principalmente para su zona de influencia y para el país, mediante mecanismos que permitan conectar espacios en redes que fortalezcan el conocimiento a través de la investigación y desarrollo de profesionales que tengan una experticia en los temas de interés que se pretendan abordar, tales como en los retos de innovación que el HUB ha planteado y ejecutado en las ciudades de influencia.

Estos retos planteados y realizados corresponden a una de las necesidades inmediatas identificadas por parte de los expertos de esta organización y a los que cada ciudad necesita dar solución con ideas innovadoras.

Las actividades realizadas corresponden a los resultados que al contar con este espacio denominado HUB Centro, se aprecia la magnitud de lo que se puede lograr con el trabajo conjunto entre las instituciones de educación superior interesadas en que los resultados de su trabajo (enseñar y facilitar el conocimiento), trasciendan más allá de sus paredes y colaboren con el mejoramiento de las condiciones de vida de su entorno, debido a que principalmente son las instituciones de educación superior miembros de esta red quienes permiten el desarrollo de estas actividades a través de sus delegados al HUB y conjuntamente con la coordinación y sus colaboradores. 


\subsubsection{Resultados}

A continuación, se abordan los resultados del HUB ITT CENTRO desarrollados desde su creación hasta los días en los que se realizó el presente artículo.

Con base en la normativa vigente aprobada, el HUB ITT CENTRO se cumplió en tres fases para la implementación del proyecto:

PRIMERA FASE: De carácter conceptual en el que se analiza, diseña y establece las condiciones para la creación del HUB. En esta fase ha sido clave el soporte técnico de la Corporación Ecuatoriana para el Desarrollo de la Investigación y la Academia (CEDIA) en la preparación del modelo de gestión, plan de inversiones, portafolio de servicios y esquema de sostenibilidad del HUB ITT CENTRO.

La fase inicial se impulsaron acuerdos para que el HUB Centro sea una realidad tal como lo señala la SENESCYT, Impulsar una articulación estratégica entre los sectores académico, social, público y productivo es el objetivo de la implementación del HUB Centro por medio de la suscripción de un acuerdo, hoy en Riobamba, con siete instituciones de educación superior [29].

Posterior a la creación del HUB Centro, se desarrollaron reuniones en las cuales se definen el modelo de gestión con el que funcionará esta organización, tal como lo mencionan CEDIA, El pasado mes de agosto en las instalaciones de CEDIA se reunieron los siete Instituciones de Educación Superior de la zonal 3 del país, con el objetivo de desarrollar un modelo de gestión para la implementación de un HUB en la región centro [30].

En este espacio de asesoramiento, CEDIA, al ser un ente imparcial direccionó las estrategias y modelos de gestión mediante un taller, el cual contó con representantes de Innovación de las IES, quienes participaron activamente buscando generar un modelo adaptable a las necesidades de la región [30].

SEGUNDA FASE: Se afinan los aspectos normativos y técnicos que hacen posible la puesta en funcionamiento del HUB ITT Centro. De forma paralela y con el apoyo directo de CEDIA, se ejecutan actividades de innovación abierta en cooperación con los GAD's Cantonales de Riobamba, Ambato y Latacunga, adicionalmente se ha tomado contacto para definir acciones con los GAD's Provinciales en la zona de influencia del HUB ITT Centro (Cotopaxi, Tungurahua y Chimborazo).

Participación en la convocatoria de la SENESCYT y de la Organización de Estados Iberoamericanos (OEI): 'Reconocimiento a las iniciativas de promoción y desarrollo de la ciencia, tecnología, innovación y saberes ancestrales de las redes del ecuador por su aporte al desarrollo socio-productivo del país' con el tema Retos de Innovación Abierta en la Zona Centro del Ecuador en la categoría proyectos I+D+i. En este evento se alcanzó una destacada participación obteniendo el segundo lugar de la categoría. 
En este encuentro nacional participaron 49 propuestas como nos detallas la SENESCYT [ ], en el evento, se premiaron a cuatro proyectos ganadores de la convocatoria Reconocimiento de las iniciativas de ciencia, tecnología e innovación y saberes ancestrales, en las categorías Difusión y divulgación y Proyectos de I+D+i, los cuales recibirán un incentivo económico [ ]. En total, se presentaron 49 propuestas, de las cuales se seleccionaron 19 para la exposición por medio de posters. Los ganadores se eligieron aplicando criterios técnicos de calidad, pertinencia, impacto y presentación.

En la categoría I+D+i, el primer lugar fue para Transporte Phenomena Research Group, con la iniciativa Acoplamiento multiescala y multifase por modelamiento y experimentación, y en segundo lugar quedó el HUB Centro, con la propuesta Retos de innovación abierta para la zona centro del Ecuador [ ].

TERCERA FASE: Se construyen alianzas estratégicas que posibiliten el crecimiento, la expansión y la sostenibilidad a largo plazo del HUB IIT Centro.

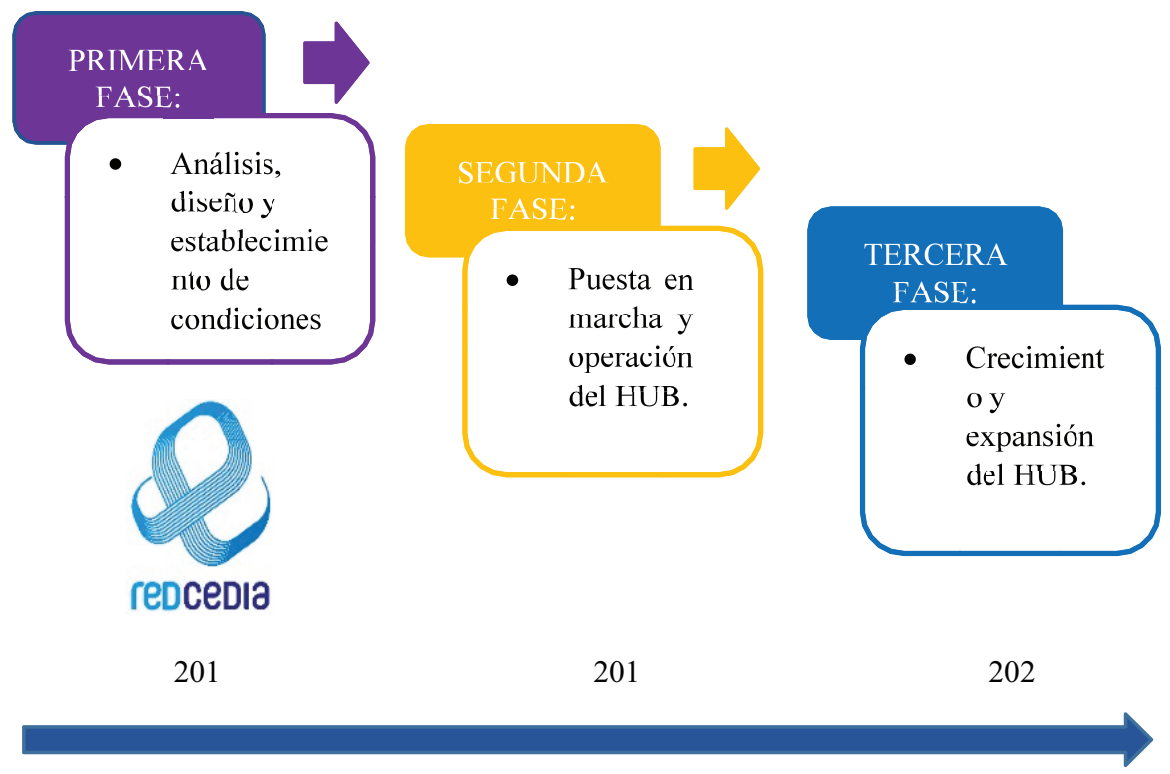

Figure 1

Fases de implementación del Proyecto HUB ITT CENTRO.

La Figura 1 presentada indica las fases en las que el proyecto HUB ITT Centro se ha realizado hasta la actualidad desde el año 2018. En esta figura se aprecian tres fases en orden cronológico de acuerdo a la gestión que esta organización ha realizado.

Entre las actividades realizadas que destacan del HUB ITT Centro tenemos las siguientes: 


\section{Conclusiones}

EI HUB ITT Centro es un espacio de innovación que impulsa a los

\section{Table 1}

Actividades ejecutadas.

\begin{tabular}{|c|c|c|}
\hline LUGAR & ACTIVIDAD & OBSERVACIÓN \\
\hline \multirow[t]{2}{*}{ Riobamba } & $\begin{array}{l}\text { Participación en la Semana de la Ciencia, } \\
\text { Tecnología, Emprendimiento e } \\
\text { Innovación SECTEl } 2019 \text { con el Reto de } \\
\text { innovación abierta - Solución a } \\
\text { problemas de transporte pùblico, } \\
\text { Riobamba. }\end{array}$ & $\begin{array}{l}\text { Se entregó } \$ 2.500 \text { al primer lugar, } \\
\text { propuesta presentada por un estudiante } \\
\text { de la ESPOCH 'Josué Cobo'. Al segundo } \\
\text { lugar se entregó } \$ 1.500, \text { propuesta } \\
\text { presentada por un profesional de Quito } \\
\text { "Diego Rosero'. }\end{array}$ \\
\hline & $\begin{array}{l}\text { Primer taller identificación de } \\
\text { problemáticas que afectan al } \\
\text { desenvolvimiento del sector productivo } \\
\text { de la provincia de Chimborazo. }\end{array}$ & $\begin{array}{l}\text { Las iniciativas priorizadas serán elevadas } \\
\text { a proyectos CEPRA y/o Fondo } 1 \text { a 1, a ser } \\
\text { financiadas por CEDIA. }\end{array}$ \\
\hline Ambato & $\begin{array}{l}\text { Taller para la definición del reto de } \\
\text { innovación abierta para la ciudad de } \\
\text { Ambato. }\end{array}$ & $\begin{array}{l}\text { Se definió el reto modelo para el } \\
\text { funcionamiento del nuevo mercado de } \\
\text { productores de la ciudad de Ambato, } \\
\text { proyecto de edificación que se } \\
\text { encuentra en la fase conceptual. }\end{array}$ \\
\hline Latacunga & $\begin{array}{l}\text { Reto de Innovación Abierta - Fomento al } \\
\text { uso de medios alternativos de transporte. }\end{array}$ & $\begin{array}{l}\text { El reto se va a realizar el } 14 \text { de noviembre } \\
\text { de } 2019 \text { a las 09:30 en el auditorio } \\
\text { Héroes del Cenepa del Campus Centro } \\
\text { de la Universidad de las Fuerzas } \\
\text { Armadas ESPE. }\end{array}$ \\
\hline
\end{tabular}

nuevos negocios o ideas de negocios para que éstos se conviertan en los generadores de desarrollo, de empleo principalmente en esta zona del país. Esta organización a través del trabajo en red busca ayudar con recursos intelectuales y de financiamiento a estos proyectos que están saliendo al mercado no solo como entidades con capacidad de contratación de recursos sino también con capacidades de mejorar el estilo de vida de las personas y satisfacer sus necesidades que son cambiantes constantemente.

Los retos realizados en las diferentes ciudades de la zona centro del país ponen al descubierto la falta de cultura social en el trabajo colaborativo para solucionar algo, se evidencia que si no existe una organización que promueva el cambio de mentalidad hacia un trabajo en equipo estos esfuerzos por alcanzar desarrollo serán en vanos, ya que tradicionalmente estamos acostumbrados a desenvolvernos en nuestro mundo y producto de ello guardar celosamente aquellos conocimientos para las mismas empresas y en muchos casos solo para las personas que realizan estas acciones.

Finalmente, el HUB siendo una organización que promueve la innovación, el emprendimiento y la transferencia de tecnología y conocimiento, y colabora con la generación de nuevas empresas, también colabora con el mejoramiento de la calidad de vida de su entorno a través de la innovación abierta que nos permite dar soluciones 
a problemas que creemos son de responsabilidad de los gobernantes. Entonces los retos realizados por el HUB nos abren la posibilidad de contribuir a resolver el impacto de estas complicaciones que vivimos a diario de una forma diferente a como estamos acostumbrados, con ideas nuevas que sean replicables en diferentes partes de acuerdo con su realidad y sobre todo que a las mejores soluciones se ofrezcan estímulos a esta manera creativa de contribuir con el progreso de la sociedad en la que vivimos.

\section{References}

[1] Palaci J. 2015. Escuela de organizacion industrial. Obtenido de Blog: https://www. eoi.es/blogs/redinnovacionEOI/2015/09/03/que-es-la-innovacion/

[2] Avendaño C, William R. Semestre económico. Redalyc. 2012. Recuperado el de: https://www.redalyc.org/articulo.oa?id=165024299008

[3] Albornoz M. Revista Iberoamericana de Ciencia, Tecnología y Sociedad - CTS. Redalyc. 2009. Retrieved from: https://www.redalyc.org/pdf/924/92415269002.pdf

[4] Anlló G, Bisang R, Campi M, Albornoz I. Comisión económica para américa latina y el caribe. Cepal. 2009. Retrieved from:: https://repositorio.cepal.org/bitstream/handle/ 11362/3652/1/S2009065_es.pdf

[5] Cámara de Comercio de España. Cámara de comercio de españa. Camara. Retrieved from: https://www.camara.es/innovacion-y- competitividad/como-innovar/tipos

[6] Innovatec. La innovación: Un factor clave para la competitividad de las empresas. Recuperado de: http://www.madrid.org/bvirtual/BVCM001260.pdf

[7] Chavez D. Banco Mundial. 2017 May 9. Recuperado de bancomundial.org: https://www.bancomundial.org/es/news/feature/2017/05/09/ innovating-for-growth-in-latin-america

[8] Rivero AC. Senior Consulting. Retrieved from: http://www.seniorconsulting.com.ar/ gestion/gestion-4-0-innovacion-es-futuro-

[9] Zapfl, D. LEAD Innovation Management. Recuperado de: https://www. lead-innovation.com/es/blog/qu\{\%\}C3\{\%\}A9-tipos-de-innovaci\{\%\}C3\{\%\}B3n-existen

[10] Ramírez MS, Peñalvo GFJ. Revista Comunicar. 2018.

[11] Benavente JM, Suaznábar C. Puntos sobre la innovación. BID. Recuperado de: https: //blogs.iadb.org/innovacion/es/politicas-de-transformacion-digital/

[12] Rodríguez LJ, Lorenzo GA. Revista galega de economía. Redalyc.org. 2010. Retrieved from: https://www.redalyc.org/pdf/391/39115737008.pdf

[13] Aros ELA, Torres BCA. Información Tecnológica. 2017.

[14] Obea Research Group. Mondragon unibertsitatea. Recuperado el: http://www. emotools.com/static/upload/files/obeainnovabrt.pdf 
[15] BBVA. Reinventar la empresa en la era digital. 2015. Recuperado el: https:/www.bbvaopenmind.com/wp-content/uploads/2015/01/ BBVA-OpenMind-libro-Reinventar-la-Empresa-en-la-Era-Digital-empresa-innovacion1-1.pdf

[16] Pombo C. Puntos sobre la innovación. 2015. Recuperado de: https://blogs.iadb.org/ innovacion/es/co-creacion-crowdsoursing-hackaton-e-innovacion-abierta-mas-que-una-moda/

[17] Sánchez B. Alianza para el Emprendimiento e Innovación. 2017 May 4. Recuperado el: https://blog.aei.ec/2017/05/04/grandes-corporaciones-impulsando-innovacion-en-ecuador/

[18] Alba Á. Blog. innolandia.es. Recuperado el: https://innolandia.es/ lanzar-retos-innovacion-enganchen/

[19] Clos I. Sociedad de la innovación. Recuperado el: https://www.sociedaddelainnovacion.es/ lo-necesitas-saber-los-retos-innovacion-elemento-clave-del-design-thinking/

[20] Centro de Ciencia y Tecnología de Antioquia - CTA. RETOS DE INNOVACIÓN MEDELLÍN. 2019. Retrieved from: http://retosinnovacionmedellin.com/ que-son-los-retos-de-innovacion/

[21] Secretaría de Educación Superior, Ciencia, Tecnología e Innovación. Banco de Ideas. 2019. Recuperado de: http://www.bancodeideas.gob.ec/convocatoria/view? data $=$ aWQ9NjA $\{\%\} 3 D$

[22] Corporación Ecuatoriana para el Desarrollo de Investigación y la Academia. CEDIA. Recuperado de: https://www.cedia.edu.ec/es/sobre-nosotros/ innovacion-y-transferencia-tecnologica/innovacion-abierta

[23] Corporación Ecuatoriana para el Desarrollo de la Investigación y la Academia - CEDIA. CEDIA. 2019. Recuperado de: https://www.cedia.edu.ec/ es/noticias-y-eventos/noticias/noticias-2019/apoyamos-la-gestion-de-hub-centro

[24] Secretaría de Educación Superior, Ciencia, Tecnología e Innovación. Educacionsuperior. Redes y Hub comparten experiencias en encuentro nacional en Ibarra. 2019. Recuperado de: https://www.educacionsuperior.gob. ec/redes-y-hub-comparten-experiencias-en-encuentro-nacional-en-ibarra/

[25] Secretaría de Educación Superior, Ciencia, Tecnología e Innovación. $\quad$ Educacionsuperior.gob.ec. 2019 March 19. Recuperado de: https://www.educacionsuperior.gob.ec/ reto-de-innovacion-abierta-el-salinerito-premia-a-sus-proyectos-ganadores/

[26] Agencia de Promociones Económicas CONQUITO. CONQUITO. Recuperado de conquito.org.ec: http://www.conquito.org.ec/wp-content/uploads/2019/10/ BRIEF-EMPRETEC.final_.2019.pdf

[27] Corpoambato. 2018. Recuperado de: http://www.corpoambato.org.ec/proyectos. html

[28] Ministerio de Producción, Comercio Exterior, Inversiones y Pesca \& CIL Centro de la Industria Láctea. Alianza para el emprendimiento e innovación. 2019 May. Recuperado de: http://www.aei.ec/desafios-de-innovacion/ 
[29] Secretaría de Educación Superior, Ciencia, Tecnología e Innovación. HUB Centro fortalece articulación entre Estado, academia y sector productivo. 2018. Recuperado de: https://www.educacionsuperior.gob.ec/ hub-centro-fortalecera-articulacion-entre-estado-academia-y-sector-productivo/

[30] Corporación Ecuatoriana para el Desarrollo de Investigación y la Academia. Desarrollamos El Modelo De Gestión Del Hub Centro. 2018. Recuperado de: $\quad$ https://www.cedia.edu.ec/es/noticias-y-eventos/noticias/noticias-2018/ desarrollamos-el-modelo-de-gestion-del-hub-centro 